\section{Patient Perspectives of Rural Kansas Maternity Care}

Meghan Blythe, M.D. ${ }^{1}$, Kathryn Istas, MPH², Shane Johnston, $\mathrm{MPA}^{3}$, Jasmine Estrada, M.D. ${ }^{1}$, Maci Hicks, M.D. ${ }^{1}$, Michael Kennedy, M.D. ${ }^{4}$

${ }^{1}$ University of Kansas School of Medicine, Kansas City, KS

${ }^{2}$ American Academy of Family Physicians, Leawood, KS

University of Kansas School of Medicine, Kansas City, KS

${ }^{3}$ Office of Medical Education

${ }^{4}$ Office of Rural Medical Education

Received Oct. 11, 2020; Accepted for publication May 5, 2021; Published online Sept. 1, 2021 https: doi.org 10.17161 limmoll414752

\section{ABSTRACT}

Introduction. Pregnant women in rural areas face a unique set of challenges due to geographic maldistribution of obstetric services. The perspectives of rural Kansas women were sought regarding experience of birth and satisfaction with maternity care.

Methods. Medical student research assistants facilitated discussion groups and structured interviews in rural Kansas communities distributed throughout the state with women who had an uncomplicated delivery in the last 24 months. Participants were recruited via convenience sampling from clinic medical records and appointments over a two-to-three-week period. Guiding questions were used to facilitate discussion. Survey instruments were used to gather information about satisfaction with maternity care. Data for qualitative and quantitative analysis was aggregated using Rural Urban Commuting Area (RUCA) codes.

Results. Fourteen groups with 47 total participants completed the survey and discussion. Participants came from large rural, small rural, and isolated areas in Kansas as described by RUCA Code Four Category Classification. Survey results indicated that satisfaction with maternity care in participants' home county was significantly higher in small rural and isolated compared to large Rural RUCAs. Qualitative analysis results showed positive experiences related to doctor characteristics, relationship with doctor, doctor's involvement with care, alternative labor options, and distance convenience. Negative experiences were related to doctor bedside manner, doctor not there until delivery, and staff related complaints.

Conclusions. Kansas women in small rural and isolated RUCA codes appeared to be more satisfied with care. Kans J Med 2021;14:220226

\section{INTRODUCTION}

Women's satisfaction with maternity services is an important measure of quality and is integral to any assessment of quality or plans for improvement of maternity care. ${ }^{1}$ Studies have suggested that quality of care is a complex concept. ${ }^{2-7}$ Many factors contributed to women's satisfaction including organization of care, resources, facilities, perceived physician concern, communication with physician and staff, perception of shared decision-making, perceived safety, and the technical competence of the practitioner.

Women in rural areas face a unique set of challenges due to a geographic maldistribution of obstetric services. ${ }^{8}$ In 2012, half of U.S. counties lacked a single obstetrician-gynecologist. Overall, the density
KANSAS JOURNAL of MEDIC INE

of obstetrician-gynecologists declined from metropolitan to micropolitan to rural counties. ${ }^{9}$ Family physicians play an important role in providing maternity care services in U.S. rural hospitals, including cesarean deliveries. ${ }^{10}$ While small rural hospitals providing maternity care services by family physicians showed no evidence of difference in perinatal outcome, ${ }^{11}$ the challenges of decreased access and increased distance to travel for maternity care have been correlated with an increase in adverse birth outcomes. ${ }^{12,13}$ The impact of rurality on maternal satisfaction has been studied previously; however, the correlations vary and there is a lack of research on rural care in America. Studies in Australia, ${ }^{14}$ New Zealand ${ }^{15}$ and Northwest Ethiopia ${ }^{16}$ showed a negative correlation between rurality and maternity care satisfaction; whereas a study from $\mathrm{Nepal}^{17}$ showed a positive correlation, and a Scottish study ${ }^{18}$ showed no correlation. Additionally, two Canadian qualitative studies reported that birth experience was influenced by geographic realities, the availability of local health services resources, and relational care characterized by time spent with the patient, continuity, and personalization. ${ }^{19-20}$

No assessment of satisfaction of maternity care has been conducted in rural Kansas, though variation in population density and population to physician ratio would suggest that differences might be found. ${ }^{21} \mathrm{We}$ hypothesized that geographical area and access to maternity care in rural and remote areas in Kansas impact the maternal birth experience and satisfaction with care.

\section{METHODS}

Study Design. Rural-Urban Commuting Areas (RUCA) were used to stratify the rurality of the maternal care experiences. The University of Washington published a four-category classification scheme using a division of the RUCA codes into urban, large rural, small rural, and isolated areas. ${ }^{22}$ Using the ERS 2010 RUCA code database ${ }^{22}$ and the University of Washington four-category classification scheme, ${ }^{23} 14$ family medicine clinics in 12 Kansas communities representing large rural, small rural, and isolated RUCAs were identified as medical student clinical rotation sites that offered maternity care with labor and delivery. Thirteen second-year medical students placed in these communities conducted the patient recruitment, informed consent, discussion, and survey facilitation at their respective site according to a standardized protocol. The family medicine clinics were a combination of private and hospital associated clinics.

Inclusion criteria included women 18 years old or older who had given birth without significant complications in the past 24 months. Patients with birth complications were excluded as it was a project goal to explain satisfaction with a typical birth experience and complications were expected to occur rarely. Patients meeting the inclusion criteria were recruited via convenience sampling and were identified from the clinic medical records and were invited via phone call using a standardized script or while in clinic for an appointment to participate in the study. No incentive was provided for participation.

This investigation employed a cross-sectional study design using a 
KANSAS JOURNAL of MEDICINE

PATIENT PERSPECTIVES OF MATERNITY CARE

continued.

combination of discussion groups/structured interviews and a single administration of a survey instrument. Groups utilized the same set of questions (Table 1), protocol, and informed consent. These guiding questions were developed from a similar focus group study assessing maternity care, ${ }^{20}$ the input of the Kansas Rural Obstetrics Access Taskforce patient and community workgroup, ${ }^{24}$ and utilized the approach to conducting a focus group described by the Mobilizing for Action through Planning and Partnerships (MAPP) Network developed by the National Association of County \& City Health Officials. ${ }^{25}$ Discussions and interviews were led according to a standardized protocol by a trained medical student research assistant and were audio recorded. Transcription was completed by a third-party and transcripts were used for qualitative analysis. Participant identifying information was not tied to the discussion comments for the purpose of anonymity.

Table 1. Guiding questions for all discussion groups.

\section{Tell us about your experience of birth.}

2. What maternity care services did you have access to in your community?

3. How satisfied were you with the services available?

4. Did you go to another community for maternity care, and if so, why?

5 . What items are important in choosing where you get maternity care?

6. If you could change things to promote better maternity care in your community, what would they be?

In addition to facilitated group discussion, participants completed a paper survey. Survey responses were recorded by research assistants. Study data were managed using the REDCap ${ }^{\circledast}$ electronic data capture tools hosted at the University of Kansas Medical Center. REDCap is a secure, web-based software platform designed to support data capture for research studies providing: 1) an intuitive interface for validated data capture; 2) audit trails for tracking data manipulation and export procedures; 3 ) automated export procedures for seamless data downloads to common statistical packages; and 4) procedures for data integration and interoperability with external sources. ${ }^{26,27}$

The survey obtained participant zip code of residence (which was used to look up the RUCA code), age, ethnicity, average annual household income, level of education, number of deliveries, miles traveled to most recent delivery, and satisfaction of maternity care in home county. These survey questions were developed from the input of the Kansas Rural Obstetrics Access Taskforce patient and community workgroup, ${ }^{24}$ and were based on bypass behavior, distance to delivery options, number of deliveries, ethnicity, age, and income. ${ }^{28}$ Participant identifying survey information was not linked to patient discussion group comments for anonymity. The University of Kansas Medical Center Institutional Review Board approved this study protocol.

Data Analysis. The primary outcome of interest was patient satisfaction of rural maternity care. This outcome was investigated using a mixed methods approach with qualitative analysis of discussion qualitative analysis began with development of the codebook of words, phrases, and patterns by two principal investigators. Each of the 14 transcripts was coded using the codebook by three team members, including two medical student research assistants previously involved in discussion group facilitation and one principal investigator. Any disagreements between the three coders were resolved by discussion. To ensure consistency, quality, and accuracy, each of the completed coded transcripts was reviewed and discussed by the entire team. Codes were added and/or combined as needed based on team consensus.

An inductive analysis of the transcripts was conducted to capture richness beyond the language constraints built into the codebook. The team discussed each transcript and compiled emerging ideas into thematic categories. Team members then pulled quotes from corresponding transcript segments to support the themes.

Quantitative analysis of survey data used Spearman correlation to measure the association between satisfaction and the following socio-demographic characteristics: RUCA code, average household income, education level, ethnicity, age, multiple pregnancies vs. first time, and miles travelled for prenatal care. Self-reported satisfaction was measured by a single item asking participants to express their level of agreement with the following statement: "I am satisfied with maternity care services in my home county". Responses were collected on a 5-point Likert scale ranging from "strongly agree" to "strongly disagree". Variables were recoded from ordered categories to numeric levels, with level 1 corresponding to the level lowest in magnitude (i.e., smallest RUCA category, least frequent, least satisfied, lowest education level, least time or distance travelled). Partial correlation measured the association between RUCA and satisfaction, controlling for distance travelled. Statistical analysis was conducted using R version 4.0.5. All analyses were based on two-tailed tests with alpha $=0.05$.

Variables for hospital in zip code, hospital with labor and delivery in zip code, hospital in county, and hospital with labor and delivery in county were derived via internet search from patient reported zip code. Patients also reported the hospital location of their most recent delivery. This was compared to the search results for hospital in county with labor and delivery and hospital in zip code with labor and delivery to derive the variables for most recent delivery in hospital with labor and delivery in county and most recent delivery in hospital with labor and delivery in zip code. Variables for county and zip code were derived and reported as zip code correlates with RUCA designation; however, many rural hospital resources were designated by county.

\section{RESULTS}

Participants. Fourteen groups with 47 total participants completed the survey and discussion. Average group size was three participants. The 14 discussion groups were held in 12 Kansas counties distributed throughout the state as shown in Figure 1. The participants were an average of 28.3 years old (range 20 - 37 years), Caucasian (96\%), and had a mean annual household income greater than $\$ 50,000$ (68\%). Participants were distributed by RUCA codes per four-category classification as follows: 17/47 (36\%) large rural, $13 / 47(28 \%)$ small rural, and 17/47 (36\%) isolated. Subject demographics are shown in Table 2. 
Table 2. Participant descriptive statistics and demographics.*

\begin{tabular}{|c|c|}
\hline Variables & Total $(\mathrm{N}=47)$ \\
\hline $\operatorname{Age}^{* *}$ & 28.3 \\
\hline $20-24$ & $11(23 \%)$ \\
\hline $25-29$ & $18(38 \%)$ \\
\hline $30-34$ & $13(28 \%)$ \\
\hline $35-49$ & $5(11 \%)$ \\
\hline \multicolumn{2}{|l|}{ Ethnicity } \\
\hline White/Caucasian & $45(96 \%)$ \\
\hline Other & $2(4 \%)$ \\
\hline \multicolumn{2}{|l|}{ Education level } \\
\hline Some high school, no diploma & $1(2 \%)$ \\
\hline High school graduate & $2(4 \%)$ \\
\hline Some college credit, no degree & $9(19 \%)$ \\
\hline Trade/technical training & $1(2 \%)$ \\
\hline Associate degree & $13(28 \%)$ \\
\hline Bachelor's degree & $18(38 \%)$ \\
\hline Master's degree & $3(6 \%)$ \\
\hline \multicolumn{2}{|l|}{ Annual household income } \\
\hline Less than $\$ 10,000$ & $1(2 \%)$ \\
\hline$\$ 10,000-\$ 14,999$ & $0(0 \%)$ \\
\hline$\$ 15,000-\$ 24,999$ & $4(9 \%)$ \\
\hline$\$ 25,000-\$ 49,999$ & $13(28 \%)$ \\
\hline$\$ 50,000$ - $\$ 99,999$ & $24(53 \%)$ \\
\hline$\$ 100,000-\$ 149,999$ & $4(9 \%)$ \\
\hline \multicolumn{2}{|l|}{ Number of births (including the birth within last 24 months) } \\
\hline 1 & $22(47 \%)$ \\
\hline 2 & $16(34 \%)$ \\
\hline 3 & $5(11 \%)$ \\
\hline 4 & $3(6 \%)$ \\
\hline 5 or more & $1(2 \%)$ \\
\hline \multicolumn{2}{|l|}{ Distance traveled for most recent delivery } \\
\hline $0-20$ miles & $37(79 \%)$ \\
\hline 20 - 40 miles & $8(17 \%)$ \\
\hline $40-60$ miles & $2(4 \%)$ \\
\hline \multicolumn{2}{|l|}{ Four-category RUCA codes } \\
\hline Large rural & $17(36 \%)$ \\
\hline Small rural & $13(28 \%)$ \\
\hline Isolated & $17(36 \%)$ \\
\hline Hospital in zip code & $32(68 \%)$ \\
\hline Hospital with labor and delivery in zip code & $30(64 \%)$ \\
\hline Hospital in county & $46(98 \%)$ \\
\hline Hospital with labor and delivery in county & $42(89 \%)$ \\
\hline Delivery at hospital with labor and delivery in zip code & $28(60 \%)$ \\
\hline Delivery at hospital with labor and delivery in county & $37(79 \%)$ \\
\hline
\end{tabular}

${ }^{*}$ Data are given as number of participants (percent of total $\mathrm{N}=47$ ) unless otherwise indicated.

**Data are given as the mean.
KANSAS JOURNAL of MEDICINE

PATIENT PERSPECTIVES OF MATERNITY CARE

continued.

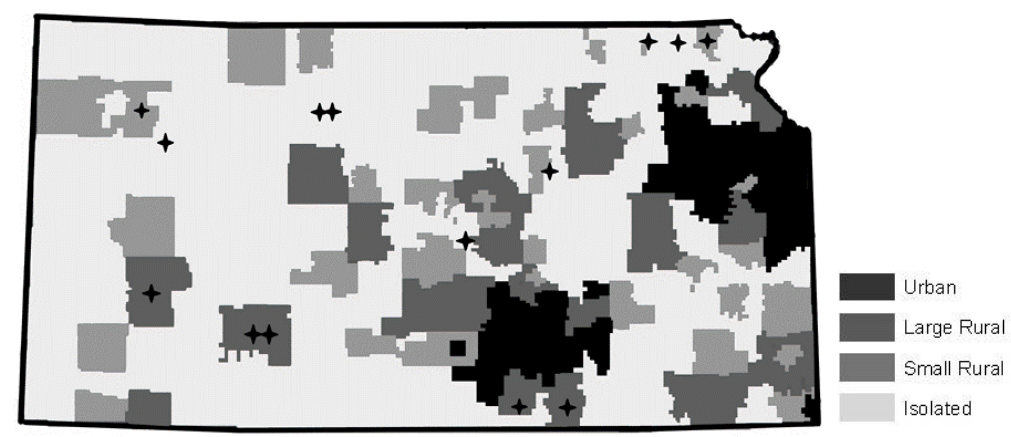

Figure 1. RUCA map of Kansas using a four-code description showing locations of discussion groups with a four-point star. ${ }^{23}$

Of the 47 participants, $32(68 \%)$ had a hospital in their zip code, 30 (64\%) had a hospital with labor and delivery in their zip code, 46 (98\%) had a hospital in their county, $42(89 \%)$ had a hospital with labor and delivery in their county, 28 (60\%) reported delivery at a hospital with labor and delivery in their zip code, and 37 (79\%) reported delivery at a hospital with labor and delivery in their county.

Quantitative Analysis. Satisfaction survey responses grouped by RUCA levels are shown in Figure 2. Higher satisfaction was associated with more rural RUCA levels $(\mathrm{r}=-0.52, \mathrm{p}<0.001)$ and with shorter distance travelled for prenatal care $(r=-0.31, \mathrm{p}<0.05)$. Higher satisfaction also was associated with more positive responses across other survey questions. For example, higher levels of satisfaction were associated with greater likelihood of delivering future children at the same place $(r=0.50, p<0.001)$ and greater likelihood of recommending the same place to another person $(r=0.36, \mathrm{p}<0.05)$.

Partial correlations were used to examine the relationship between RUCA level and satisfaction after accounting for the relationship between proximity to a hospital and satisfaction. The partial correlation between RUCA level and satisfaction was $\mathrm{r}=-0.48(\mathrm{p}<0.001)$ after adjusting for whether respondents had a hospital offering obstetrical services in their county or zip code. The partial correlation between RUCA level and satisfaction was $r=-0.36(p<0.05)$ when further adjusting for level of agreement with the statement, "I will go for obstetrical services wherever I have the most confidence in the hospital and facilities whether this is in my county or not".

Qualitative Analysis. A range of one to six participants was included per discussion group/structured interview with an average of three participants per discussion group. Using the codebook, discussion group participants' stories of their birth experiences (responses to Question 1) were coded as positive or negative and responses to Questions 2 - 6 were categorized accordingly.

The mention of themes during the participant discussion groups/ structured interviews, shown in Table 3, provided insight into the difference of participant satisfaction by rurality. The codes were binary and counted if the theme was mentioned during the discussion/interview. This avoided a group of more participants having more cumulative codes. 
KANSAS JOURNAL of MEDICINE

PATIENT PERSPECTIVES OF MATERNITY CARE

continued.
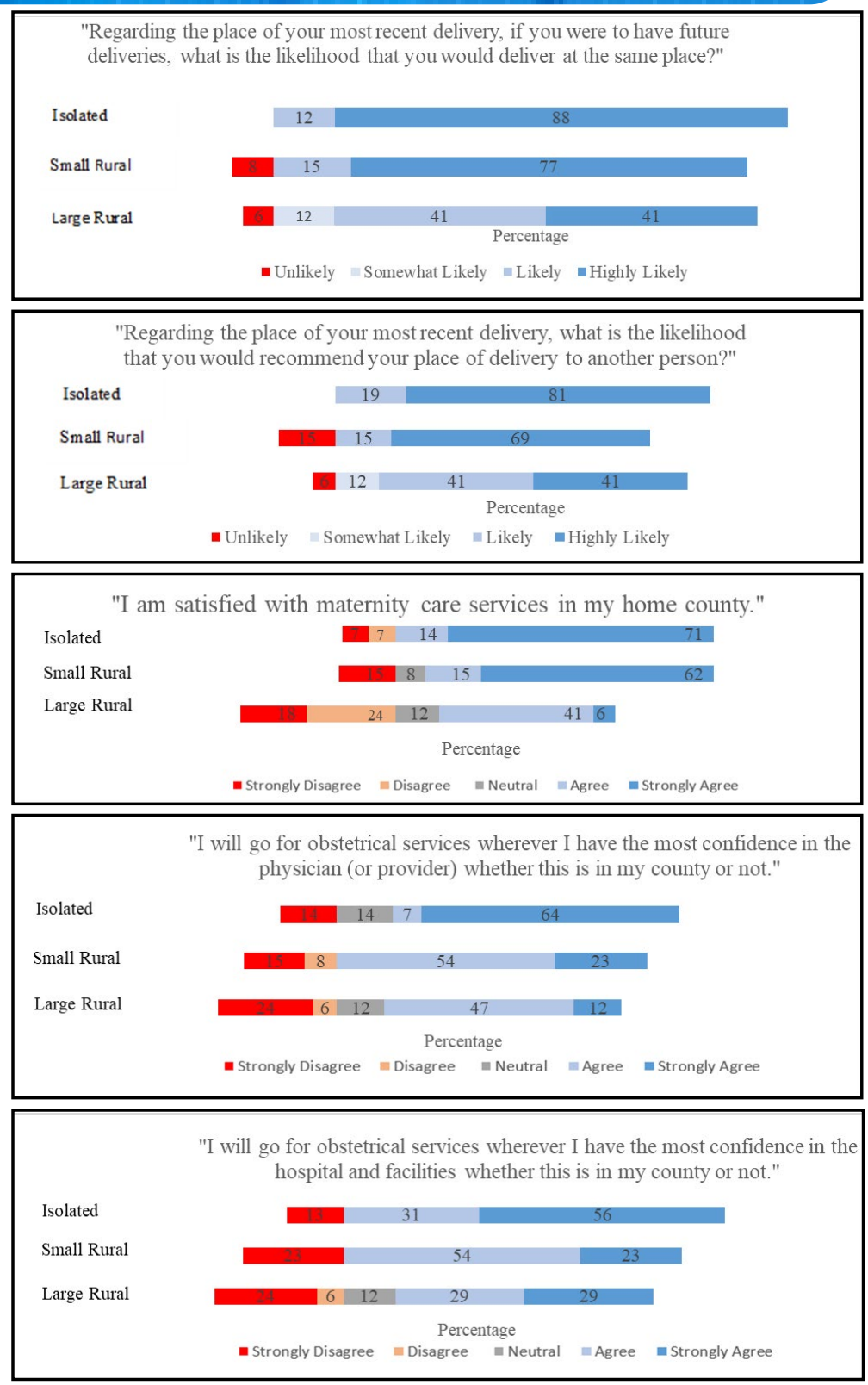

Figure 2. Satisfaction survey responses grouped by RUCA Code $(n=47)$.

More isolated discussion groups mentioned positive birth experiences related to their doctor being family medicine trained, details about their hospital room, and other positive birth experiences that included personal attention from staff and immediate skin to skin contact with the baby. Participant quotes from isolated discussion groups supporting these themes included:

- "[The doctor] is my general practitioner as well so they knew [my past medical history]. So they took care of me the whole time and would monitor my pregnancy and other conditions; that really did help."

- "I like the really big windows in my room. The natural light made such a big difference because I was exhausted but wasn't disoriented."

- "The personal attention really helped; I was the only person delivering in the hospital."
More small rural and isolated discussion groups mentioned positive birth experiences related to convenience. Participant quotes from isolated and small rural discussion groups supporting the theme of convenience included:

- "[The hospital location] was very convenient. My doctor told me that if my water was to break naturally, then I would have time to shower and get something to eat. I had plenty of time for all of that. Had I not lived in the same town [as the hospital] I'm not sure if I would have taken the time to shower and eat something before I left. I probably would have been a little bit more freaked out of having to drive 30 minutes to the nearest hospital."

- "I was very glad that we were only 10 minutes away [from the hospital] because I think I had 4 contractions in the car and I don't think I could have done too many more because my baby was born soon after got to the hospital."

Additionally, more large rural discussion groups mentioned negative birth experiences related to their doctor. Poor bedside manner, doctor not there until delivery, and delivery with a different doctor were sub-themes identified. Participant quotes from large rural discussion groups supporting the theme of doctor related negative birth experiences included:

- "[The doctor] had really bad bedside manners and the whole experience was just miserable."

- "I thought the doctor should have been checking on me the whole time I was in the hospital, but she didn't come until the very last minute."

•"I had my baby on a weekend, and my doctor wasn't there. So I had a different doctor who I did not care for at all."

Themes identified as being "Factors Important in Choosing Care" are shown in Table 4. Across discussion groups/structured interviews held in large rural, small rural, and isolated Kansas RUCAs, more isolated discussion groups mentioned family medicine trained and doctor characteristics as factors that were important to their choice of care. Participant quotes from isolated discussion groups supporting these themes included:

- "I preferred a family doctor. [The doctor] can be your OB, your doctor, and your kid's doctor. So everything."

- "[The doctor] is relatable and understands my lifestyle and religious beliefs."

More small rural and isolated discussion groups mentioned convenience as being important to choosing maternity care. Participant quotes supporting the theme of convenience included:

- "I work full time and didn't want to take off half a day to doctor in the neighboring county."

- "My husband is a farmer and busy certain times of year; that puts me on my own if I need to come in fast so I wanted to deliver somewhere close." 
Table 3. Mention of themes by four-category RUCA in response to Question 1 (Tell us about your experience of birth).

\begin{tabular}{|c|c|c|c|c|c|}
\hline & & $\begin{array}{l}\text { Four-category RUCA by zip code of discussion } \\
\text { group location }\end{array}$ & Large rural & Small rural & Isolated \\
\hline & Theme & Sub-theme (examples) & $\mathbf{n}=\mathbf{4}$ & $\mathbf{n}=\mathbf{5}$ & $\mathbf{n}=\mathbf{5}$ \\
\hline \multirow{7}{*}{ Positive experience } & \multirow[t]{2}{*}{ Doctor related } & $\begin{array}{l}\text { Doctor characteristics (available, open, makes me } \\
\text { laugh, supportive, communication/bedside manner, } \\
\text { trust/confidence, trust/respect of other doctors, par- } \\
\text { ent/doctor has kids too) }\end{array}$ & 4 & 3 & 5 \\
\hline & & Family medicine trained/"general practitioner" & 2 & - & 3 \\
\hline & \multicolumn{2}{|c|}{ Staff related (OB nurse, nurse, midwife, lactation consultant) } & 4 & 3 & 5 \\
\hline & Resource related & Room related (own room, natural light) & 1 & 1 & 3 \\
\hline & Distance related & Convenience & 1 & 3 & 4 \\
\hline & \multicolumn{2}{|l|}{ Medical related (VBAC, epidural) } & - & 2 & 1 \\
\hline & \multicolumn{2}{|c|}{ Other (personal attention, immediate skin to skin) } & - & - & 3 \\
\hline \multirow{5}{*}{ Negative experience } & Doctor related & & 4 & 1 & 1 \\
\hline & Staff related & & 2 & 2 & 3 \\
\hline & Distance related & & - & 1 & 1 \\
\hline & $\begin{array}{l}\text { Medical related (Gestational } \\
\text { Diabetes, nausea, epidural, } \\
\text { induction) }\end{array}$ & & 1 & 1 & 3 \\
\hline & $\begin{array}{l}\text { Other (billing confusion, lack } \\
\text { of communication with referral } \\
\text { center) }\end{array}$ & & - & 2 & 1 \\
\hline
\end{tabular}

Table 4. Mention of themes by four-category RUCA in response to Question 5 (What items are important in choosing where you get maternity care?).

\begin{tabular}{|c|c|c|c|c|c|}
\hline & & $\begin{array}{l}\text { Four-category RUCA by zip code of discussion } \\
\text { group location }\end{array}$ & Large rural & Small rural & Isolated \\
\hline & Theme & Sub-theme (examples) & $\mathbf{n}=\mathbf{4}$ & $\mathbf{n}=\mathbf{5}$ & $\mathbf{n}=\mathbf{5}$ \\
\hline \multirow{9}{*}{$\begin{array}{l}\text { Factors important in } \\
\text { choosing care }\end{array}$} & \multirow{4}{*}{ Doctor related } & $\begin{array}{l}\text { Doctor characteristics (comfortable with doctor, } \\
\text { bedside manner, character, personality, credibility, } \\
\text { trust, relatable) }\end{array}$ & 1 & 2 & 4 \\
\hline & & Family medicine trained & 1 & 1 & 3 \\
\hline & & Female provider & 1 & 1 & 1 \\
\hline & & Personal relationship with doctor & - & 1 & 1 \\
\hline & \multirow[t]{2}{*}{ Distance related } & Doctor response time & - & 2 & 1 \\
\hline & & Convenience & 1 & 5 & 5 \\
\hline & \multicolumn{2}{|c|}{$\begin{array}{l}\text { Resource related factor (labor options, doula, birthing class, VBAC, lactation consulta- } \\
\text { tion) }\end{array}$} & - & 1 & 1 \\
\hline & \multicolumn{2}{|c|}{ Recommendation from others/reputation } & 2 & 2 & 1 \\
\hline & \multicolumn{2}{|c|}{ Patient works at hospital/clinic } & 1 & 3 & 1 \\
\hline
\end{tabular}


KANSAS JOURNAL of MEDICINE

PATIENT PERSPECTIVES OF MATERNITY CARE

continued.

\section{DISCUSSION}

Key Findings. The quantitative analysis of survey results suggested that rurality influences satisfaction of maternity care, specifically that women in small rural and isolated Kansas RUCA codes appeared to be more satisfied with care. From qualitative analysis of the discussions/ interviews, these results seemed to be a factor of convenience of having a local maternity care option, as well as influenced by doctor characteristics and family medicine training.

As many rural counties rely on maternity care provided by family physicians, ${ }^{10}$ we expected mention of family medicine trained doctors during the discussions/interviews. For isolated discussion groups, the theme of family physicians was identified as a doctor related positive experience, as well as a factor important to choosing care. Additional comments linked to mention of care by a family physician include "relationship with doctor", "delivery with my doctor", and "prior experience with doctor". These results highlighted the broad scope of practice including maternity care offered by family physicians.

Female provider was identified from the discussions as a factor influencing maternity care decisions. This theme was not mentioned in previous studies regarding satisfaction of rural care, nor was it captured in the survey results. The influence of this theme in rural Kansas warrants further investigation.

The derived variables of hospital in zip code, hospital with labor and delivery in zip code, hospital in county, and hospital with labor and delivery in county suggested a disproportion of facilities that did not offer labor and delivery. This disproportion has been described as "obstetrical deserts" ${ }^{24}$ Despite the existence of this disproportion in our study, the majority of participants were satisfied with their maternity care.

When compared to the derived variables of hospital with labor and delivery in zip code and hospital with labor and delivery in county, the derived variables of delivery at hospital with labor and delivery in zip code and delivery at hospital with labor and delivery in county showed a difference of 2 (4\%) and 5 (11\%), respectively. Further investigation is needed to explain these differences, but it may represent "bypass behavior" or a patient's choice to seek services other than those offered in their home county. ${ }^{28}$

Implications and Application of Findings. The identified themes and codebook will inform future research questions and projects, including a clinic survey of women in rural areas regarding satisfaction of care and distance traveled.

Study Limitations. The number of participants was not reflective of the more than 9,853 births in rural Kansas counties during the time of the study; there were a total of 36,264 live births statewide. ${ }^{29}$ The selection of study participants was through general solicitation and participants were not selected randomly. However, there was no specific selective process to get participants. Overall, the convenient sampling method made the results subject to general bias. Participant recruitment was not bilingual and may have contributed to under sampling of
Hispanic populations in rural Kansas. Additional bias is acknowledged as some participants reported working for or having worked at the local clinic and/or hospital. There was, however, good representation across the state by region and RUCA code.

\section{CONCLUSIONS}

Women in small rural and isolated RUCA codes in Kansas appeared to be more satisfied with their maternity care. From the discussions/ interviews, these results seemed to be a factor of the convenience of a local maternity care option as well as doctor characteristics and family medicine training.

\section{ACKNOWLEDGEMENTS}

The authors acknowledge and thank the participating patients in rural Kansas communities and the medical student research assistants who collected data for this project. Abstracts of these results were presented at the KUMC Student Research Forum on April 1, 2019 (Kansas City, KS), the National Rural Health Association Annual Conference May 7-9, 2019 (Atlanta, GA), and the Kansas Academy of Family Physicians Annual Conference on June 8, 2019 (Wichita, KS). This project was supported in part by a KUMC Auxiliary grant. There was also general funding for the STORM program from the Hansen Foundation, Sutton Fund, KAFP-Foundation, and the Baker Trust.

\section{REFERENCES}

${ }^{1}$ Perriman N, Davis D. Measuring maternal satisfaction with maternity care: A systematic integrative review: What is the most appropriate, reliable and valid tool that can be used to measure maternal satisfaction with continuity of maternity care? Women Birth 2016; 29(3): 293-299. PMID: 26782087.

${ }^{2}$ Lewis L, Hauck YL, Ronchi F, Crichton C, Waller L. Gaining insight into how women conceptualize satisfaction: Western Australian women's perception of their maternity care experiences. BMC Pregnancy Childbirth 2016; 16:29. PMID: 26846257.

${ }^{3}$ Zweig S, Kruse J, LeFevre M. Patient satisfaction with obstetric care. J Fam Pract 1986; 23(2):131-136. PMID: 3734717.

4 Attanasio L, McPherson ME, Kozhimannil KB. Positive childbirth experiences in US hospitals: A mixed methods analysis. Matern Child Health J 2014; 18(5):1280-1290. PMID: 24072597.

${ }^{5}$ Goberna-Tricas J, Banús-Giménez MR, Palacio-TausteA, Linares-Sancho S. Satisfaction with pregnancy and birth services: The quality of maternity care services as experienced by women. Midwifery 2011; 27(6):e231-237. PMID: 21145632.

${ }^{6}$ Séguin L, Therrien R, Champagne F, Larouche D. The components of women's satisfaction with maternity care. Birth 1989; 16(3):109-113. PMID: 2789558.

7 Handler A, Raube K, Kelley MA, Giachello A. Women's satisfaction with prenatal care settings: A focus group study. Birth 1996 ;23(1):31-37. PMID: 8703255 .

${ }^{8}$ Rayburn WF, Richards ME, Elwell EC. Drive times to hospitals with perinatal care in the United States. Obstet Gynecol 2012;119(3):611-616. PMID: 22353960.

9 Rayburn WF, Klagholz JC, Murray-Krezan C, Dowell LE, Strunk AL. Distribution of American Congress of Obstetricians and Gynecologists fellows and junior fellows in practice in the United States. Obstet Gynecol 2012; 119(5):1017-1022. PMID: 22525913.

${ }^{10}$ Young R. Maternity care services provided by family physicians in rural hospitals. J Am Board Fam Med 2017; 30(1): 71-77. PMID: 28062819.

${ }^{11}$ Chaska BW, Mellstrom MS, Grambsch PM, Nesse RE. Influence of site of obstetric care and delivery on pregnancy management and outcome. J Am Board Fam Pract 1988; 1(3):152-163. PMID: 3177013.

${ }^{12}$ Grzybowski S, Stoll K, Kornelsen J. Distance matters: A population based study examining access to maternity services for rural women. BMC Health Serv Res 2011; 11(1):147-155. PMID: 21663676.

${ }^{13}$ Larimore WL, Davis A. Relation of infant mortality to the availability of maternity care in rural Florida. J Am Board Fam Pract 1995; 8(5):392-399. PMID: 7484227.

14 Brown S, Lumley J. Satisfaction with care in labor and birth: A survey of 790 Australian women. Birth 1994; 21(1):4-13. PMID: 8155224. 
15 Dawson P, Hay-Smith J, Jaye C, Gauld R, Auvray B. Do maternity services in New Zealand's public healthcare system deliver on equity? Findings from structural equation modelling on national maternal satisfaction survey data. Midwifery 2021; 95:102936. PMID: 33592369.

${ }^{16}$ Gashaye KT, Tsegaye AT, Shiferaw G, Worku AG, Abebe SM. Client satisfaction with existing labor and delivery care and associated factors among mothers who gave birth in university of Gondar teaching hospital; Northwest Ethiopia: Institution based cross-sectional study. PLoS One 2019; 14(2):e0210693. PMID: 30726297.

17 Mehata S, Paudel YR, Dariang M, et al. Factors determining satisfaction among facility-based maternity clients in Nepal. BMC Pregnancy Childbirth 2017; 17(1):319. PMID: 28946851.

${ }^{18}$ Cheyne H, Elders A, Hill D, Milburn E. Is maternity care in Scotland equitable? Results of a national maternity care survey. BMJ Open 2019; 9(2):e023282. PMID: 30804027.

19 Sutherns R. Adding women's voices to the call for sustainable rural maternity care. Can J Rural Med 2004; 9(4):239-244. PMID: 15603695.

${ }^{20}$ Kornelsen J, Grzybowski S. The reality of resistance: The experiences of rural parturient women. J Midwifery Womens Health 2006; 51(4):260-265. PMID: 16814220

${ }^{21}$ County Health Rankings. 2020 County Health Rankings. State Reports. http://www.countyhealthrankings.org. Accessed March 20, 2018.

${ }^{22}$ USDA Economic Research Service. Rural-Urban Commuting Area Codes. https://www.ers.usda.gov/data-products/rural-urban-commutingarea-codes.aspx. Accessed March 8, 2018.

${ }^{23}$ WWAMI Rural Health Research Center. RUCA Four category classification, Census Division. https://depts.washington.edu/uwruca/ruca-maps. php. Accessed March 8, 2018.

${ }^{24}$ Kansas Rural Obstetric Access Task Force. Kansas Rural Obstetrics Access Task Force Report and Recommendations, 2017. Unpublished document, 2017.

${ }^{25}$ National Association of County and City Health Officials. Mobilizing for Action through Planning and Partnerships (MAPP). https://www.naccho. org/programs/public-health-infrastructure/performance-improvement/ community-health-assessment/mapp. Accessed March 20, 2018.

${ }^{26}$ Harris PA, Taylor R, Thielke R, Payne J, Gonzalez N, Conde JG. Research electronic data capture (REDCap)--a metadata-driven methodology and workflow process for providing translational research informatics support. J Biomed Inform 2009; 42(2):377-381. PMID: 18929686.

${ }^{27}$ Harris PA, Taylor R, Minor BL, et al. The REDCap consortium: Building an international community of software platform partners. J Biomed Inform 2019; 95:103208. PMID: 31078660.

${ }^{28}$ Liu JJ, Bellamy GR, McCormick M. Patient bypass behavior and critical access hospitals: Implications for patient retention. J Rural Health 2007; 23(1):17-24. PMID: 17300474.

29 Mosier S. Annual Summary of Kansas Vital Statistics, 2016. Kansas Department of Health and Environment. September 2017. http://www. kdheks.gov/phi/as/2016/Annual_Summary_2016.pdf. Accessed March 8, 2018.

Keywords: obstetrics, rural health services, patient satisfaction, Kansas
KANSAS JOURNAL of MEDICINE

PATIENT PERSPECTIVES OF MATERNITY CARE continued. 